\title{
BMJ Open Increase in the oxidised low-density lipoprotein level by smoking and the possible inhibitory effect of statin therapy in patients with cardiovascular disease: a retrospective study
}

\author{
Kazuo Ogawa, Toshikazu Tanaka, Tomohisa Nagoshi, Hiroshi Sekiyama, \\ Satoshi Arase, Kosuke Minai, Takayuki Ogawa, Michihiro Yoshimura
}

To cite: Ogawa K, Tanaka T, Nagoshi T, et al. Increase in the oxidised low-density lipoprotein level by smoking and the possible inhibitory effect of statin therapy in patients with cardiovascular disease: a retrospective study. BMJ Open 2015;5: e005455. doi:10.1136/ bmjopen-2014-005455

- Prepublication history for this paper is available online. To view these files please visit the journal online (http://dx.doi.org/10.1136/ bmjopen-2014-005455).

Received 12 April 2014 Revised 16 November 2014 Accepted 19 December 2014

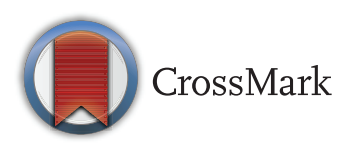

Division of Cardiology, Department of Internal Medicine, The Jikei University School of Medicine, Tokyo, Japan

Correspondence to Dr Kazuo Ogawa; oga-n@jikei.ac.jp

\section{ABSTRACT}

Objectives: Malondialdehyde-modified low-density lipoprotein (MDA-LDL) level is a marker of oxidative stress and is linked to progression of arteriosclerosis; however, the clinical factors affecting the oxidised LDL level have not been elucidated. We investigate various factors to identify correlation with MDA-LDL level in high-risk patients requiring catheter intervention.

Setting: Secondary care (cardiology), single-centre study.

Participants: 600 patients who were admitted to our hospital and underwent cardiac catheterisation.

Primary and secondary outcome measures:

Blood samples were obtained to measure lipid profiles and MDA-LDL level.

Results: With regard to smoking status, MDA-LDL level was significantly higher in ex-smokers/current smokers compared with non-smokers. Of note, there was no improvement of MDA-LDL level even in patients who had quit smoking. Multiple regression analysis showed that MDA-LDL level was positively correlated with LDL-cholesterol (LDL-C) level, Brinkman index and male gender. The correlation between smoking status and either MDA-LDL or LDL-C level was investigated in two groups: namely, patients with and patients without statin treatment. In the nonstatin group, MDA-LDL level and MDA-LDL/LDL-C ratio were significantly higher in ex-smokers/current smokers compared with non-smokers, while no significant correlation was observed between smoking status and LDL-C level. In contrast, in the statin group, there were no significant correlations between smoking status and any of the cholesterol parameters.

Conclusions: We found that MDA-LDL level was affected by multiple factors, such as smoking status, LDL-C level and male gender. The present findings give additional evidence that smoking should be prohibited from a MDALDL standpoint. Furthermore, statin therapy might have a beneficial effect on the reduction of MDA-LDL level.

\section{INTRODUCTION}

The malondialdehyde modified low-density lipoprotein (MDA-LDL; oxidised LDL) is
Strengths and limitations of this study

- Although oxidative low-density lipoprotein (LDL) is associated with the marker of oxidative stress and progression of atherosclerosis, the clinical factor affecting the oxidative LDL remains uncertain. Our study revealed that malondialdehydemodified (MDA)-LDL was associated with smoking and MDA-LDL level would never decrease with smoking cessation. However, MDA-LDL level was decreasing even in smokers with statin therapy.

- Smoking cessation was not found to be effective for reducing MDA-LDL level in this study; however, favourable effects of smoking cessation would likely occur with regard to parameters other than MDA-LDL level. Thus, smoking cessation is recommended at any time, even after long-term smoking, and is considered to provide cardiovascular health benefits.

- This was a retrospective study, and the true effect of statin on MDA-LDL level remains uncertain. Finally, we did not examine the prognosis of the study population and, therefore, the effect of smoking cessation and/or statin therapy remains uncertain, especially in terms of their impact on MDA-LDL level. Prospective studies are required to obtain answers regarding these topics.

LDL that has been modified by MDA, leading to the production of a large amount of aldehyde when LDL becomes degenerated and oxidised. ${ }^{1}$

It is known that MDA-LDL level is elevated in patients with dyslipidaemia and diabetes mellitus (DM), both risks factors for atherosclerotic disease. ${ }^{23}$ Since MDA-LDL level has a positive correlation with the serum LDL level, the ratio of MDA-LDL/LDL-cholesterol (LDL-C; M/L) is used to evaluate the severity of oxidisation of LDL; in some reports, not only MDA-LDL level, but also $\mathrm{M} / \mathrm{L}$ ratio has 
been shown to increase in patients with DM compared with controls. ${ }^{45}$

In patients with coronary artery disease (CAD), MDA-LDL level and $\mathrm{M} / \mathrm{L}$ ratio have been shown to increase even when there are no other differences in the other lipid profiles. ${ }^{6}$ In addition, it has been shown that the measurement of MDA-LDL level might be useful as a predictor of restenosis after percutaneous coronary intervention in patients with $\mathrm{DM}^{7}{ }^{7}$ Based on these findings, it has been speculated that MDA-LDL level might be an important marker of the progression of arteriosclerosis; however, the clinical factors possibly affecting MDA-LDL level have not been elucidated. Therefore, in the present study, we investigated 'the clinical factors' affecting MDA-LDL level in high-risk patients requiring catheter intervention.

\section{METHODS}

Study patients

Six hundred consecutive patients who underwent cardiac catheterisation from March 2010 to September 2011 were examined in this study. The baseline patient characteristics, including clinical parameters and biochemical data, were collected retrospectively from hospital medical records. In addition, the results of the catheterisation (ie, the number of occluded or narrowed vessels), body weight, body mass index (BMI), coronary risk factors and medication profiles were also investigated. Patients taking eicosapentaenoic acid (EPA) were excluded since it has been demonstrated that EPA is a major lipid-lowering agent with potent antioxidant effects. We complied with the routine ethical regulation of our institution as follows. This is a retrospective study and informed consent could not be obtained from each patient. Instead of informed consent from each patient, we publicly posted a notice about the study design and contact information at a publicly known space in our institution.

\section{Data collection}

Blood sampling was performed to examine the serum MDA-LDL, serum creatinine ( $\mathrm{Cr}$ ), glycated haemoglobin (HbA1c), B-type (brain) natriuretic peptide (BNP) and LDL-C levels. The previous study reported by Tsimikas et $a l^{8}$ contended that percutaneous coronary intervention (PCI) would affect the oxidative LDL level. To avoid the modification of LDL level by PCI, we tried to take a blood draw immediately before the cardiac catheterisation or at the outpatient clinic before admission. MDA-LDL level was measured by an ELISA using an anti-MDA-LDL monoclonal antibody (ML25) and $\beta$-galactosidase anti-apoB monoclonal antibody (AB16). ${ }^{1}$ It is well known that the combination of ML25 and AB16 can accurately detect MDA-LDL. ${ }^{1}$ The concentration of MDA-LDL is defined at $1 \mathrm{mg} / \mathrm{L}$ of MDA-LDL produced artificially, which shows the same signal as $1 \mathrm{U} / \mathrm{L}$ of MDA-LDL in the serum. Serum levels of
LDL-C were determined enzymatically (Sekisui Medical Co, Ltd, Tokyo, Japan).DM, hypertension and smoking were defined as described previously. ${ }^{9}{ }^{10}$ Dyslipidaemia was diagnosed with the use of lipid-lowering agents, the presence of one or more of the following three lipid disorders at first fasting blood sampling, or both: a LDL-C level $\geq 140 \mathrm{mg} / \mathrm{dL}$, a triglyceride level $\geq 150 \mathrm{mg} / \mathrm{dL}$ and a high-density lipoprotein (HDL) cholesterol (HDL-C) level $<40 \mathrm{mg} / \mathrm{dL} .{ }^{9}$ Blood sampling was performed on the day of the catheter examination, except for the cases for which it had already been performed at outpatient clinic. Among 600 patients, 342 blood samplings were performed on the day of the catheter examination, and the remaining 258 were performed at outpatient clinic.

The definition of smoking status is as follows: current smokers were those who were smoking at the time of the study or who had smoked in the past year; the participants who had quit smoking more than 1 year before the study were defined as ex-smokers and those who had never smoked were defined as non-smokers. Brinkman index was used to evaluate the smoking status of current smokers/ex-smokers. ${ }^{11}$

\section{Statistical analysis}

Comparisons between MDA-LDL level and LDL-C level, age, BMI, HbAlc, Cr, BNP and Brinkman index were performed with a linear regression analysis. Comparison between Brinkman index and LDL-C was also performed with a linear regression analysis. Comparisons of MDA-LDL level between males and females, participants with or without hypertension, non-smokers versus ex-smokers versus current smokers, and among the various patient groups after smoking cessation, were performed with Mann-Whitney U test. Kruskal-Wallis test was performed to evaluate the difference between MDA-LDL (C) and LDL-C (D) among four groups divided by Brinkman index. Multiple factors that were considered to possibly modify MDA-LDL levels were evaluated with a stepwise multiple regression analysis. Comparison of LDL-C and MDA-LDL level, and M/L ratio between non-smokers and smokers was performed with Mann-Whitney U test.

A value of $p<0.05$ was considered to be statistically significant for all data that were statistically analysed using the SPSS software package, V.21.0 (SPSS Inc, Chicago, Illinois, USA).

\section{RESULTS}

\section{Baseline characteristics}

The baseline characteristics of the patients in this study were shown in table 1 . The average age was 64.8 \pm 11.4 years and $80.3 \%$ were male. The percentages of non-smokers, ex-smokers and current smokers were $32 \%, 42 \%$ and $26 \%$, respectively. The average LDL-C and MDA-LDL levels were $106.1 \pm 30.8 \mathrm{mg} / \mathrm{dL}$ and 119.2 $\pm 48.7 \mathrm{U} / \mathrm{L}$, respectively. The percentage of patients taking statin therapy was $55.3 \%$. In addition, patient 
characteristics divided by smoking status into three groups (non-smokers, ex-smokers, current smokers) are shown in table 2.

\section{Clinical factors affecting MDA-LDL level}

To elucidate the determinants of MDA-LDL level, a simple regression analysis was performed (figure 1). MDA-LDL level showed a significantly positive correlation with LDL-C level (figure 1A) and a negative correlation with age (figure 1B). In addition, MDA-LDL level was significantly higher in male as well as patients without hypertension (figure 1C, D). BMI, HbA1c, Cr and BNP level had no impact on MDA-LDL level (figures 1E-H).

\section{Correlation of smoking status with MDA-LDL level}

Next, the impact of smoking status on MDA-LDL level was performed. MDA-LDL, but not LDL-C, showed a significantly positive correlation with the smoking profiles

\begin{tabular}{|c|c|}
\hline $\mathrm{N}=600$ & Mean \pm SD \\
\hline Age (years) & $64.8 \pm 11.4$ \\
\hline Male, gender (\%) & 80.3 \\
\hline Height $(\mathrm{cm})$ & $165.4 \pm 25.1$ \\
\hline Weight (kg) & $66.8 \pm 13.3$ \\
\hline $\mathrm{BMI}\left(\mathrm{kg} / \mathrm{m}^{2}\right)$ & $24.4 \pm 3.74$ \\
\hline Non-smoker, N (\%) & $192(32.4)$ \\
\hline Ex-smoker, $\mathrm{N}(\%)$ & $247(41.7)$ \\
\hline Current smoker, N (\%) & $153(25.8)$ \\
\hline $\mathrm{Cr}(\mathrm{mg} / \mathrm{dL})$ & $1.38 \pm 1.94$ \\
\hline eGFR $\left(\mathrm{mL} / \mathrm{min} / 1.73 \mathrm{~m}^{2}\right)$ & $62.6 \pm 21.6$ \\
\hline HbA1c (\%) & $6.4 \pm 1.1$ \\
\hline BNP (pg/mL) & $140 \pm 263$ \\
\hline LDL-C (mg/dL) & $106.1 \pm 30.8$ \\
\hline MDA-LDL (U/L) & $119.2 \pm 48.7$ \\
\hline \multirow[t]{2}{*}{$\mathrm{M} / \mathrm{L}$} & $1.16 \pm 0.47$ \\
\hline & $\mathrm{N}(\%)$ \\
\hline \multicolumn{2}{|l|}{ Disease } \\
\hline Diabetes mellitus & $252(42.0)$ \\
\hline Hypertension & $455(75.5)$ \\
\hline Dyslipidaemia & $441(73.5)$ \\
\hline \multicolumn{2}{|l|}{ Medicine } \\
\hline Statin & $332(55.3)$ \\
\hline Ezetimibe & $31(5.2)$ \\
\hline Fibrate & $13(2.1)$ \\
\hline \multicolumn{2}{|l|}{ Coronary artery disease } \\
\hline OVD & $215(35.8)$ \\
\hline 1VD & $249(41.5)$ \\
\hline 2VD & $84(14.0)$ \\
\hline 3VD & $52(8.7)$ \\
\hline
\end{tabular}

OVD, 0-vessel disease; 1VD, single-vessel disease; 2VD, double-vessel disease; 3VD, triple-vessel disease.

$\mathrm{BMI}$, body mass index; BNP, B-type natriuretic peptide; $\mathrm{Cr}$, creatinine; eGFR, estimated glomerular filtration rate; $\mathrm{HbA1c}$, glycated haemoglobin; LDL-C, low-density lipoprotein cholesterol; $\mathrm{M} / \mathrm{L}$, malondialdehyde-modified low-density lipoprotein/low-density lipoprotein cholesterol; MDA-LDL, malondialdehyde-modified low-density lipoprotein. indicated by Brinkman index (figure 2A, B). However, considering the bias of the Brinkman distribution, in addition to that shown in figure 2A, B, MDA-LDL and LDL-C levels were analysed dividing by Brinkman index into four groups. There was a significant difference of MDA-LDL level between non-smoker and current smoker, whereas there was no significant difference of LDL-C regardless of the smoking status (figure $2 \mathrm{C}, \mathrm{D}$ ).

The patients were subsequently divided into three groups according to their smoking status: non-smokers, ex-smokers and current smokers. MDA-LDL level was significantly higher in ex-smokers and current smokers compared with that in non-smokers (figure 2E). Moreover, when patients were compared based on the number of years after smoking cessation, not only the current smoking group, but also the group that had quit smoking within 10 years and where the patients had quit smoking more than 21 years earlier showed higher MDA-LDL levels than did the non-smoking group (figure 2F).

\section{Clinical factors affecting MDA-LDL level identified in multiple regression analysis}

To assess the independent determinants of MDA-LDL level, a multiple regression analysis was performed. After removing the confounding factors, MDA-LDL level was shown to be positively correlated with LDL-C level $(\mathrm{p}<0.001)$, Brinkman index $(\mathrm{p}=0.009)$ and male gender $(\mathrm{p}=0.019$; table 3$)$.

\section{Effects of statin therapy on the correlation of smoking status with MDA-LDL or LDL-C level}

The correlation of smoking status with MDA-LDL/LDL-C level was investigated after patients were divided into two groups: those with and those without statin treatment (figure 3). In the non-statin-treated group, MDA-LDL level as well as $\mathrm{M} / \mathrm{L}$ ratio were significantly increased in ex-smokers/current smokers compared with nonsmokers, although there was no significant difference in LDL-C level between the participants with different smoking status. LDL-C level was not significantly different in non-statin-treated and statin-treated groups.

\section{DISCUSSION}

In this study, we investigated the factors associated with MDA-LDL level in high-risk patients requiring cardiac catheterisation. According to a multivariate analysis, Brinkman index, as well as the LDL-C level and gender, were found to be significantly associated with MDA-LDL level. Furthermore, we found that smoking cessation was not effective for reducing MDA-LDL level, even after the patients had quit smoking for many years. However, we found evidence that statin treatment may reduce MDA-LDL level, which could possibly help in the treatment of smokers.

It has been reported that smoking may affect susceptibility of plasma LDL to peroxidative modification. 
Table 2 Patient characteristics divided by smoking status

\begin{tabular}{|c|c|c|c|}
\hline & Non-smoker & Ex-smoker & Current smoker \\
\hline Number of patients (\%) & $192(32.4)$ & $250(42.2)$ & $151(25.4)$ \\
\hline Age & $66.9 \pm 12.4$ & $65.5 \pm 9.5$ & $60.9 \pm 12.2$ \\
\hline Male, gender (\%) & $110(57.3)$ & $228(91.2)^{\star}$ & $138(91.4)$ \\
\hline Height $(\mathrm{cm})$ & $160.3 \pm 11.0$ & $166.0 \pm 11.8^{*}$ & $167.4 \pm 7.3$ \\
\hline Weight (kg) & $62.7 \pm 14.0$ & $67.7 \pm 10.9^{\star}$ & $70.6 \pm 14.8$ \\
\hline BMI $\left(\mathrm{kg} / \mathrm{m}^{2}\right)$ & $24.2 \pm 3.6$ & $24.3 \pm 3.0$ & $25.0 \pm 4.9$ \\
\hline $\mathrm{Cr}(\mathrm{mg} / \mathrm{dL})$ & $1.2 \pm 1.5$ & $1.6 \pm 2.1$ & $1.3 \pm 2.1$ \\
\hline $\mathrm{HbA} 1 \mathrm{c}(\%)$ & $6.3 \pm 0.9$ & $6.4 \pm 1.1$ & $6.4 \pm 1.1$ \\
\hline BNP (pg/mL) & $167.1 \pm 302.0$ & $133.2 \pm 264.8$ & $120.7 \pm 201.1$ \\
\hline LDL-C (mg/dL) & $102.3 \pm 29.1$ & $104.2 \pm 28.4$ & $114.8 \pm 35.2 \dagger$ \\
\hline MDA-LDL (U/L) & $108.0 \pm 43.6$ & $122.1 \pm 49.2^{*}$ & $129.3 \pm 52.1$ \\
\hline $\mathrm{M} / \mathrm{L}$ & $2.06 \pm 0.85$ & $2.26 \pm 0.82$ & $2.6 \pm 0.99$ \\
\hline LVEF (\%) & $60.2 \pm 10.0$ & $56.7 \pm 9.6^{\star}$ & $54.4 \pm 12.1$ \\
\hline Diabetes mellitus & $72(37.5)$ & $112(44.8)$ & $65(43.0)^{\star}$ \\
\hline Hypertension & $155(80.7)$ & $191(76.4)$ & $104(68.9)^{\star}$ \\
\hline Dyslipidaemia & $134(69.8)$ & $185(74.0)$ & $116(76.8)$ \\
\hline Statin & $111(57.8)$ & $146(58.4)$ & $73(48.3)$ \\
\hline Ezetimibe & $10(3.6)$ & $14(5.6)$ & $7(4.6)$ \\
\hline Fibrate & $2(1.0)$ & $6(2.4)$ & $5(3.3)$ \\
\hline \multicolumn{4}{|l|}{ Coronary artery disease } \\
\hline OVD & $82(42.7)$ & 83 (33.2) & $49(32.5)$ \\
\hline 1VD & 69 (35.9) & $105(42.0)$ & $71(47.0)^{*}$ \\
\hline 2VD & $26(13.5)$ & $34(42.0)$ & $22(14.6)$ \\
\hline 3VD & $15(7.8)$ & $28(7.2)$ & $9(6.0)$ \\
\hline \multicolumn{4}{|c|}{$\begin{array}{l}{ }^{*} \mathrm{p}<0.05 \text { versus non-smoker. } \\
+\mathrm{tp}<0.05 \text { versus ex-smoker. } \\
\text { OVD, } 0 \text {-vessel disease; } 1 \mathrm{VD} \text {, single-vessel disease; } 2 \mathrm{VD} \text {, double-vessel disease; } 3 \mathrm{VD} \text {, triple-vessel disease; BMI, body mass index; BNP, } \\
\text { B-type natriuretic peptide; Cr, creatinine; HbA1c, glycated haemoglobin; LDL-C, low-density lipoprotein cholesterol; LVEF, left ventricular } \\
\text { ejection fraction; M/L, malondialdehyde-modified low-density lipoprotein/low-density lipoprotein cholesterol; MDA-LDL, } \\
\text { malondialdehyde-modified low-density lipoprotein. }\end{array}$} \\
\hline
\end{tabular}

Modified LDL has been shown to be the preferred substrate for macrophages and induces their subsequent transformation into foam cells. ${ }^{12} 13$ Thus, oxidative stress is very important for synthesis of modified-LDL. However, it is noteworthy that MDA-LDL level was emphatically influenced by smoking but not by obesity
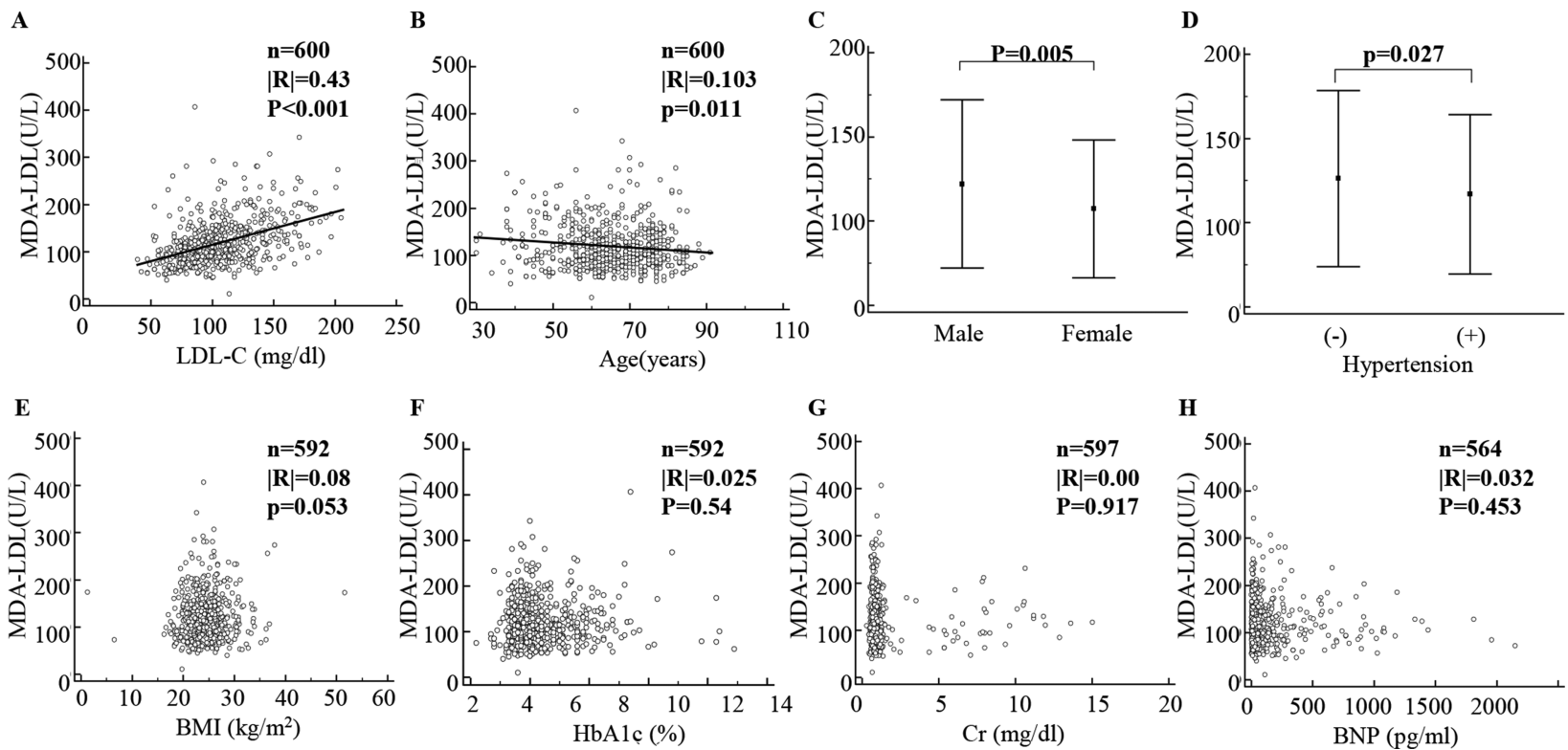

Figure 1 Correlations of MDA-LDL level with various clinical factors. The clinical factors affecting MDA-LDL level are shown in (A-H). MDA-LDL, malondialdehyde-modified low-density lipoprotein; LDL-C, low-density lipoprotein cholesterol; BMI, body mass index; $\mathrm{HbA1c}$, glycated haemoglobin; $\mathrm{Cr}$, creatinine; BNP, B-type natriuretic peptide. 
A

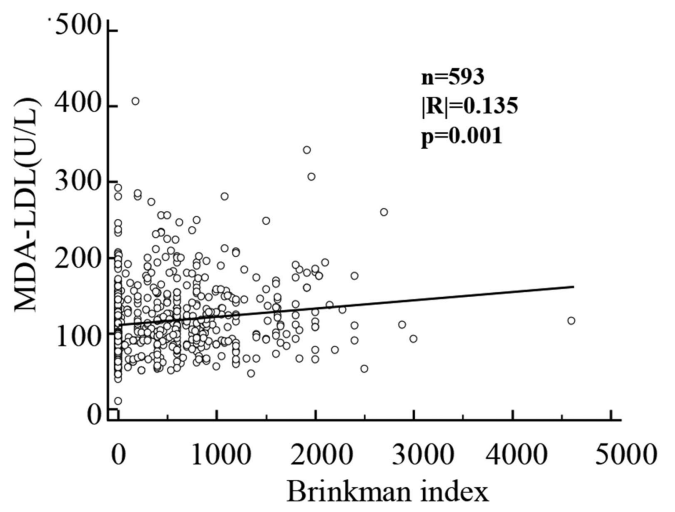

C

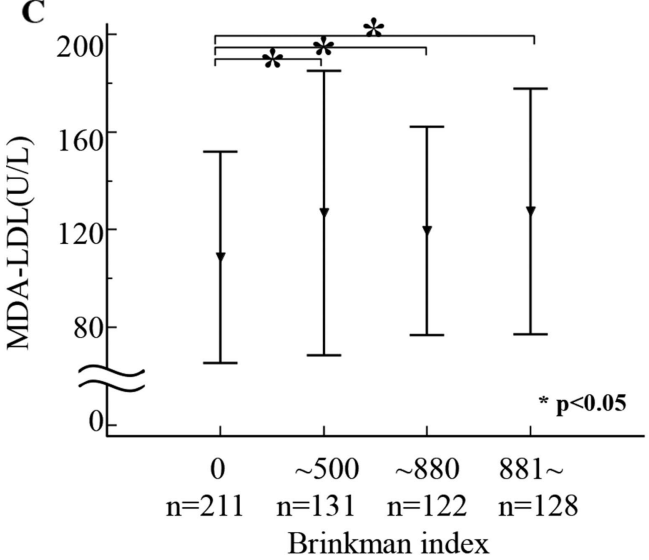

E

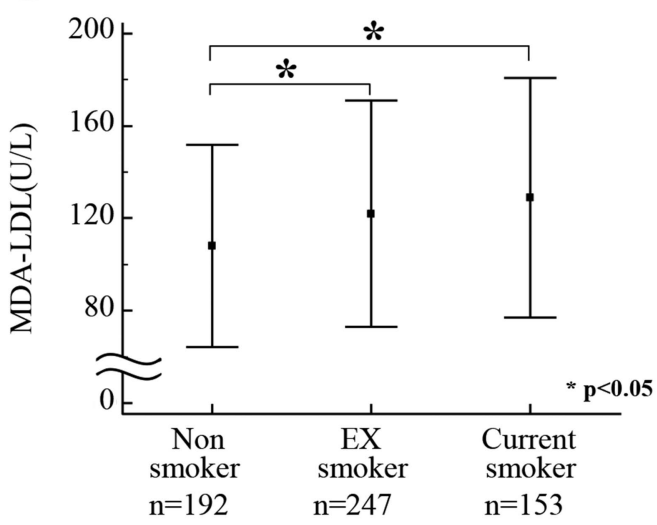

B

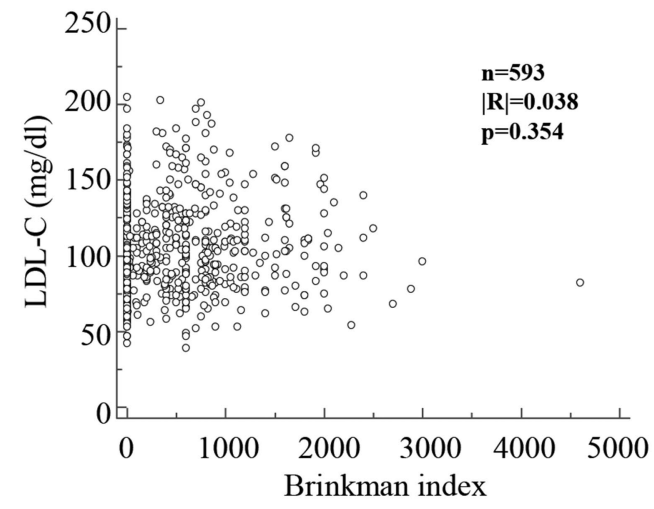

D

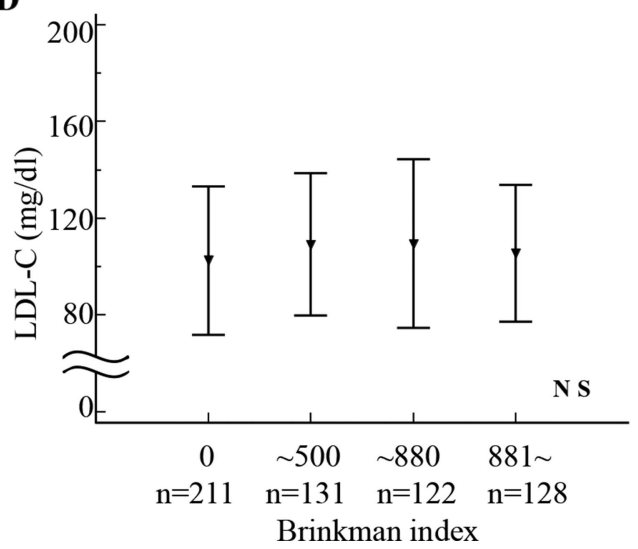

F

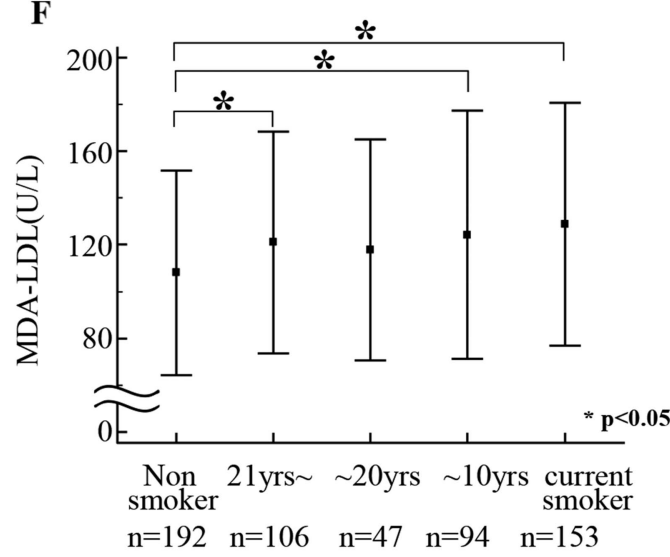

Duration After Cessation(yrs=years)

Figure 2 Correlation of smoking status with malondialdehyde-modified low-density lipoprotein (MDA-LDL) and low-density lipoprotein cholesterol (LDL-C) levels. Correlation between MDA-LDL level and Brinkman index (A), and between LDL-C level and Brinkman index (B) was determined with linear regression analysis. Kruskal-Wallis test was performed to evaluate the difference between MDA-LDL (C) and LDL-C (D) among 4 groups divided by Brinkman index. Comparison of MDA-LDL levels among non-smokers, ex-smokers and current smokers (E). Comparison of MDA-LDL levels in each patient group among non-smokers and ex-smokers who had quit more than 21 years earlier, ex-smokers who had quit 11-20 years ago, ex-smokers who had quit less than 10 years ago and current smokers (F).

(estimated by BMI), hypertension, DM (estimated by HbA1c level), renal failure (estimated by Cr level) or heart failure (estimated by BNP level) by the multivariate analysis in this study, though all of these clinical characteristics have been shown to increase oxidative stress. The precise mechanism by which smoking increases MDA-LDL level remains unclear at present.
The current study clearly showed that smoking was substantially harmful with regard to increasing MDA-LDL level. When we examined the effect of smoking status on MDA-LDL level among non-smokers, ex-smokers and current smokers, we found that MDA-LDL level was still higher in ex-smokers than in non-smokers and was unexpectedly similar to the level in current smokers. 
Table 3 Multiple regression analysis

\begin{tabular}{llllr}
\hline $\begin{array}{l}\text { Significant } \\
\text { variable }\end{array}$ & $\begin{array}{l}\text { Regression } \\
\text { coefficients }\end{array}$ & SE & $\begin{array}{l}\text { Standard regression } \\
\text { coefficients }\end{array}$ & F \\
\hline LDL-C & 0.675 & 0.06 & 0.429 & 128.089 \\
Brinkman index & 0.008 & 0.003 & 0.105 & 71.502 \\
Gender & 11.511 & 4.908 & 0.94 & 49.901 \\
\hline
\end{tabular}

Objective variable: MDA-LDL.

Explanatory variable: BMI, age, gender, Brinkman index, Cr, BNP, LDL-C, HbA1c, HTN.

No significant variables: BMI, age, Cr, BNP, HbA1c, HTN.

BMI, body mass index; BNP, B-type natriuretic peptide; Cr, creatinine; HbA1c, glycated haemoglobin; HTN, hypertension; LDL-C, low-density

lipoprotein cholesterol; MDA-LDL, malondialdehyde-modified low-density lipoprotein.

Furthermore, we examined the effects of the period of smoking cessation and the analysis indicated that even many years after smoking cessation, there was no significant reduction of MDA-LDL level. This result suggests that smoking should never be started, and that if started, it is important to quit smoking as soon as possible before becoming a heavy smoker.

The present result suggests that smoking keeps MDA-LDL level elevated for a long time. Therefore, a method for lowering MDA-LDL level is especially needed for smokers. One possibility might be statin therapy. Statins facilitate the LDL uptake in hepatocytes, decrease old LDL (which is easily oxidised), and thereby reduce the risk of LDL oxidisation. ${ }^{14}$ The decreases in fatty acids and cholesterol in the lipoprotein are also likely to lead to a decrease in oxidisation. ${ }^{15}$ In the current study, we examined the effect of statin treatment on MDA-LDL level between non-smokers and smokers. MDA-LDL level was found to be significantly higher in smokers than in non-smokers in the statin (-) group. On the other hand, the effect of smoking on LDL-C level was not seen in the statin $(+)$ group, the level was similar between smokers and non-smokers. The M/L ratio showed a similar result. These results may suggest that statin therapy would reduce MDA-LDL level in smokers to a level similar to that in non-smokers.

It has been reported that cigarette smoking is one of the risk factors for organic stenosis but that it does not act alone in contributing to the progression of atherosclerosis. ${ }^{15} 16$ In addition, atherosclerosis was not produced by smoking alone in animal models. ${ }^{17}$ However, cigarette smoking acts in concert with other risk factors
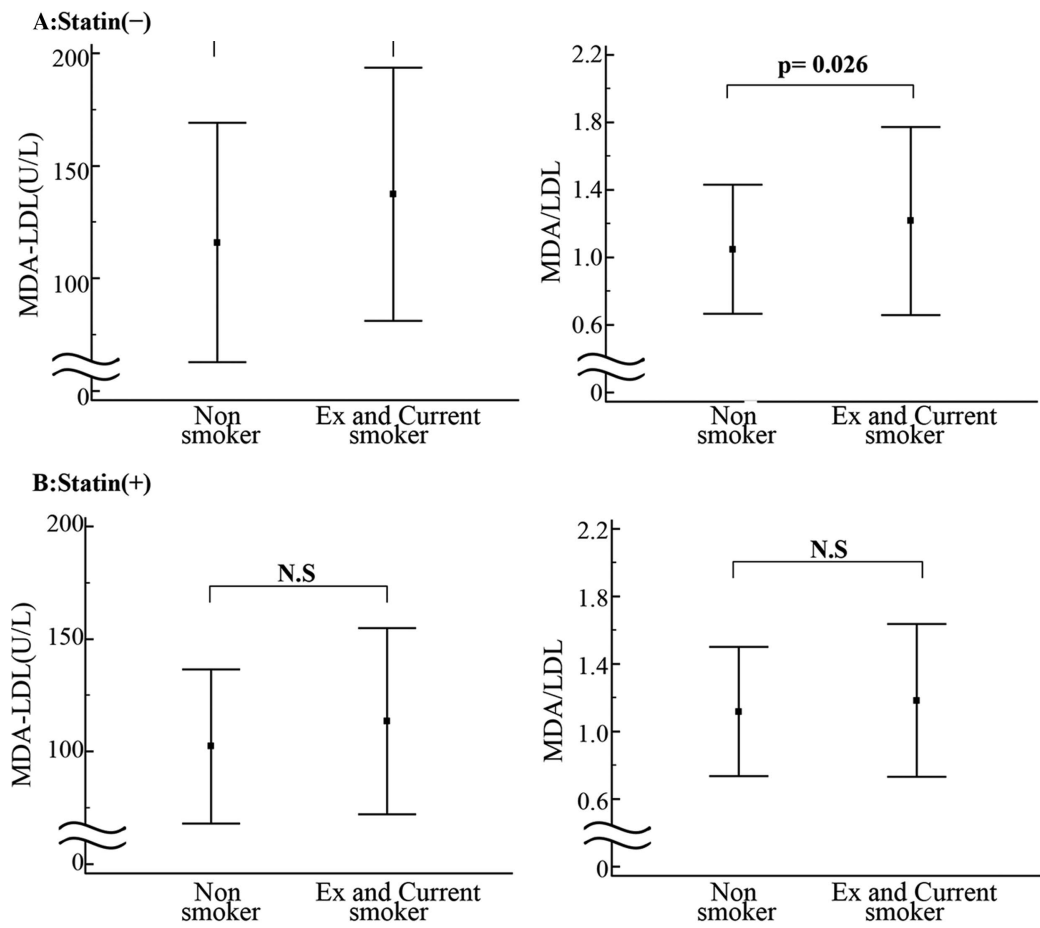

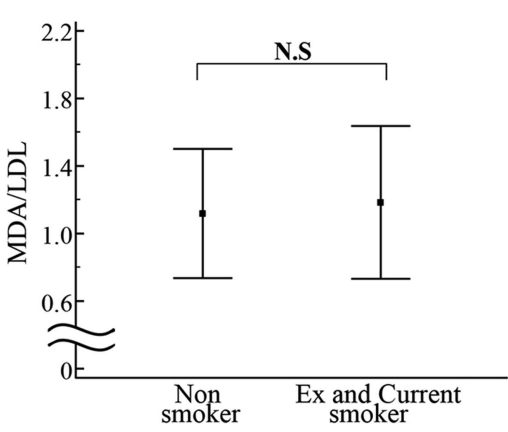

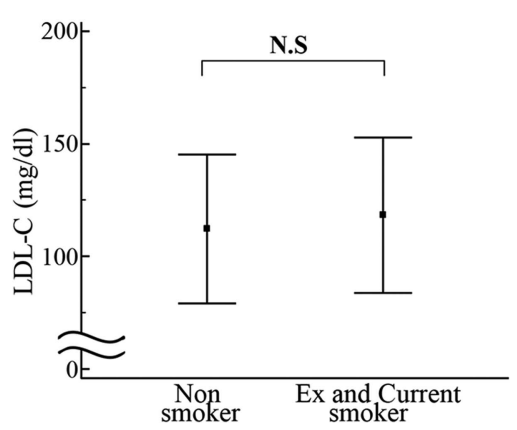

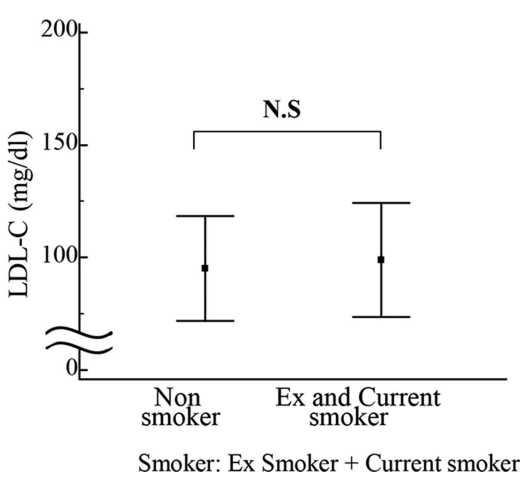

Figure 3 Effects of statin therapy on the correlations of smoking status with malondialdehyde-modified low-density lipoprotein (MDA-LDL) or low-density lipoprotein cholesterol (LDL-C) level. The correlations of smoking status with MDA-LDL and LDL-C levels were investigated after dividing the patients into two groups; with (A) or without (B) statin treatment. 
such as hypercholesterolaemia to accelerate atherosclerosis. $^{14-1618} 19$ The current study is in agreement with the previous reports. Importantly, it has been shown that among other risk factors, cigarette smoking alone is a highly significant risk factor for coronary spasm, ${ }^{20-24}$ and treatment with fluvastatin reduced the coronary spasm. ${ }^{25}$ Thus, smoking cessation combined with statin therapy would be beneficial for prevention of ischaemic heart disease by reducing progression of atherosclerosis and suppressing coronary spasm.

In the present study, we performed multivariate analysis for determination of MDA-LDL by using the factors of LDL-C level, age, BMI, HbAlc, Cr, BNP and Brinkman index; however, there may be other factors associated with MDA-LDL that act in a direct or indirect manner. As a matter of fact, the previous study by Matsuda $e t a l^{26}$ showed that MDA-LDL level was correlated with triglyceride, HDL-C, metformin and $\alpha$-glucosidase inhibitors in patients with statin-treated diabetes with CAD. Then, we performed another multivariate analysis. As a result, it revealed that MDA-LDL level was correlated with LDL-C, triglyceride and smoking, however, it was not correlated with HDL-C, antihypertensive drugs, antidiabetes agents such as metformin and $\alpha$-glucosidase inhibitors, and number of vessels with $\mathrm{CAD}$ and $\mathrm{G}$ reactive protein (precise data not shown). Furthermore, there may be confounding factors among them; and the correlation among each factor was also investigated. As a result, there was only slight correlation between $\mathrm{CAD}$ and HDL-C ( $\mathrm{R}=$ $-0.163)$, between $\mathrm{CAD}$ and smoking $(\mathrm{R}=0.098)$, between triglyceride and HDL-C ( $\mathrm{R}=0.203)$, and between triglyceride and smoking $(\mathrm{R}=-0.163$; precise data not shown). The reason for the difference between the previous study and ours may be due to the different study population. In any case, it would be safe to say that smoking affected MDA-LDL level in a fairly direct manner.

\section{CONCLUSION}

We found that MDA-LDL level was affected by multiple factors such as smoking status (as indicated by Brinkman index), LDL-C level and gender. In addition to its other health effects, smoking should be strongly forbidden due to its harmful effect from a MDA-LDL standpoint. We recommend that patients should never smoke, but if smoking has started, it is essential to quit smoking as early as possible or to cut back on the number of cigarettes consumed. Furthermore, statin therapy might have a beneficial effect on the reduction of MDA-LDL level.

Contributors $\mathrm{KO}, \mathrm{TT}, \mathrm{KM}$ and $\mathrm{MY}$ conceived of and designed the experiments. $\mathrm{KO}, \mathrm{HS}, \mathrm{SA}, \mathrm{TN}$ and TO performed the experiments. KO, HS, KM and MY performed the statistical analysis. KO, TT, TN and KM made reagents/ materials/analysis tools. KO, TT, KM and MY wrote the paper.

Funding This research received no specific grant from any funding agency in the public, commercial or not-for-profit sectors.

Competing interests None.

Ethics approval The study protocol (24-150[6916]) was approved by the ethics committee of the Jikei University School of Medicine.
Provenance and peer review Not commissioned; externally peer reviewed.

Data sharing statement No additional data are available.

Open Access This is an Open Access article distributed in accordance with the Creative Commons Attribution Non Commercial (CC BY-NC 4.0) license, which permits others to distribute, remix, adapt, build upon this work noncommercially, and license their derivative works on different terms, provided the original work is properly cited and the use is non-commercial. See: http:// creativecommons.org/licenses/by-nc/4.0/

\section{REFERENCES}

1. Kotani K, Maekawa M, Kanno T, et al. Distribution of immunoreactive malondialdehyde-modified low-density lipoprotein in human serum. Biochim Biophys Acta 1994;1215:121-5.

2. Kitano S, Kanno T, Maekawa M, et al. Improved method for the immunological detection of malondialdehyde-modified low-density lipoproteins in human serum. Anal Chim Acta 2004;509:229-35.

3. Kondo A, Manabe M, Saito K, et al. Insulin treatment prevents LDL from accelerated oxidation in patients with diabetes. $J$ Atheroscler Thromb 2002;9:280-7.

4. Kondo A, Muranaka Y, Ohta I, et al. Relationship between triglyceride concentrations and LDL size evaluated by malondialdehyde-modified LDL. Clin Chem 2001;47:893-900.

5. Kondo A, Li J, Manabe M, et al. Relationship between high-density lipoprotein-cholesterol and malondialdehyde-modified low-density lipoprotein concentrations. J Atheroscler Thromb 2003;10:72-8.

6. Tanaga K, Bujo H, Inoue M, et al. Increased circulating malondialdehyde-modified LDL levels in patients with coronary artery diseases and their association with peak sizes of LDL particles. Arterioscler Thromb Vasc Biol 2002;22:662-6.

7. Shigematsu S, Takahashi N, Hara M, et al. Increased incidence of coronary in-stent restenosis in type 2 diabetic patients is related to elevated serum malondialdehyde-modified low-density lipoprotein. Circulation J2007;71:1697-702.

8. Tsimikas S, Lau HK, Han KR, et al. Percutaneous coronary intervention results in acute increases in oxidized phospholipids and lipoprotein(a): short-term and long-term immunologic responses to oxidized low-density lipoprotein. Circulation 2004;109:3164-70.

9. Yagi H, Komukai K, Hashimoto $\mathrm{K}$, et al. Difference in risk factors between acute coronary syndrome and stable angina pectoris in the Japanese: smoking as a crucial risk factor of acute coronary syndrome. J Cardiol 2010;55:345-53.

10. Sekiyama H, Nagoshi T, Komukai K, et al. Transient decrease in serum potassium level during ischemic attack of acute coronary syndrome: paradoxical contribution of plasma glucose level and glycohemoglobin. Cardiovasc Diabetol 2013;12:4.

11. Brinkman GL, Coates EO Jr. The effect of bronchitis, smoking, and occupation on ventilation. Am Rev Respir Dis 1963;87:684-93.

12. Yokode $\mathrm{M}$, Kita $\mathrm{T}$, Arai $\mathrm{H}$, et al. Cholesteryl ester accumulation in macrophages incubated with low density lipoprotein pretreated with cigarette smoke extract. Proc Natl Acad Sci USA 1988;85:2344-8.

13. Harats $D$, Ben-Naim $M$, Dabach $Y$, et al. Cigarette smoking renders LDL susceptible to peroxidative modification and enhanced metabolism by macrophages. Atherosclerosis 1989;79:245-52.

14. Gotto AM Jr. Interactions of the major risk factors for coronary heart disease. Am J Med 1986;80:48-55.

15. Willett WC, Green A, Stampfer MJ, et al. Relative and absolute excess risks of coronary heart disease among women who smoke cigarettes. N Engl J Med 1987;317:1303-9.

16. Stein $Y$, Harats $D$, Stein $O$. Why is smoking a major risk factor for coronary heart disease in hyperlipidemic subjects? Ann N Y Acad Sci 1993;686:66-9.

17. Asano M, Ohkubo C, Hirokawa A, et al. Smoking Research Foundation Annual Research Report, on macro- and microcirculatory effects of tobacco smoke inhalation on atherogenesis in the rabbit. 1987:251-61.

18. Anderson KM, Wilson PW, Odell PM, et al. An updated coronary risk profile. A statement for health professionals. Circulation 1991;83:356-62.

19. Holbrook JH, Grundy SM, Hennekens $\mathrm{CH}$, et al. Cigarette smoking and cardiovascular diseases. A statement for health professionals by a task force appointed by the steering committee of the American Heart Association. Circulation 1984;70:1114a-17a.

20. Caralis DG, Deligonul U, Kern MJ, et al. Smoking is a risk factor for coronary spasm in young women. Circulation 1992;85:905-9.

21. Sugiishi M, Takatsu F. Cigarette smoking is a major risk factor for coronary spasm. Circulation 1993;87:76-9. 
22. Yoshimura M, Yasue H, Nakayama M, et al. A missense Glu298Asp variant in the endothelial nitric oxide synthase gene is associated with coronary spasm in the Japanese. Hum Genet 1998;103:65-9.

23. Nakayama M, Yasue $\mathrm{H}$, Yoshimura M, et al. T-786-->C mutation in the 5'-flanking region of the endothelial nitric oxide synthase gene is associated with coronary spasm. Circulation 1999;99:2864-70.

24. Takaoka K, Yoshimura M, Ogawa $\mathrm{H}$, et al. Comparison of the risk factors for coronary artery spasm with those for organic stenosis in a Japanese population: role of cigarette smoking. Int J Cardiol 2000;72:121-6.
25. Yasue $\mathrm{H}$, Mizuno $\mathrm{Y}$, Harada $\mathrm{E}$, et al. Effects of a

3-hydroxy-3-methylglutaryl coenzyme A reductase inhibitor,

fluvastatin, on coronary spasm after withdrawal of calcium-channel blockers. J Am Coll Cardiol 2008;51:1742-8.

26. Matsuda M, Tamura R, Kanno K, et al. Impact of dyslipidemic components of metabolic syndrome, adiponectin levels, and anti-diabetes medications on malondialdehyde-modified low-density lipoprotein levels in statin-treated diabetes patients with coronary artery disease. Diabetol Metab Syndr 2013; $5: 77$. 